\title{
Common and Distinct Mechanisms of Cognitive Flexibility in Prefrontal Cortex
}

\author{
Chobok Kim, ${ }^{1}$ Nathan F. Johnson, ${ }^{1}$ Sara E. Cilles, ${ }^{1}$ and Brian T. Gold ${ }^{1,2}$ \\ ${ }^{1}$ Department of Anatomy and Neurobiology, and ${ }^{2}$ Magnetic Resonance Imaging and Spectroscopy Center, University of Kentucky, Lexington, Kentucky \\ 40536
}

The human ability to flexibly alternate between tasks represents a central component of cognitive control. Neuroimaging studies have linked task switching with a diverse set of prefrontal cortex (PFC) regions, but the contributions of these regions to various forms of cognitive flexibility remain largely unknown. Here, subjects underwent functional brain imaging while they completed a paradigm that selectively induced stimulus, response, or cognitive set switches in the context of a single task decision performed on a common set of stimuli. Behavioral results indicated comparable reaction time costs associated with each switch type. Domain-general task-switching activation was observed in the inferior frontal junction and posterior parietal cortex, suggesting core roles for these regions in switching such as updating and representing task sets. In contrast, multiple domain-preferential PFC activations were observed across lateral and medial PFC, with progressively more rostral regions recruited as switches became increasingly abstract. Specifically, highly abstract cognitive set switches recruited anterior-PFC regions, moderately abstract response switches recruited mid-PFC regions, and highly constrained stimulus switches recruited posterior-PFC regions. These results demonstrate a functional organization across lateral and medial PFC according to the level of abstraction associated with acts of cognitive flexibility.

\section{Introduction}

A remarkable capacity of the human cognitive control system involves the ability to switch between multiple tasks (Miller and Cohen, 2001). Neuroimaging studies of cognitive control have identified diverse prefrontal cortex (PFC) regions that contribute to task switching (for review, see Sakai, 2008). The diverse set of $\mathrm{PFC}$ regions recruited suggests that the brain may house multiple switching mechanisms. Behavioral studies have recognized at least three switch types: stimulus (or perceptual), response, and cognitive set switching (Meiran and Marciano, 2002). Stimulus switching involves switching between stimulus selection rules or perceptual-stimulus associations (e.g., switching between circle and square according to a selection rule). Response switching refers to switching between different response rules or opposing stimulus-response (S-R) mappings (e.g., either triangle-left button and rectangle-right button or vice versa). Cognitive set (or set) switching involves shifting between task rules or sets (e.g., the Wisconsin card sorting task).

The existence of multiple, distinct switch mechanisms likely contributes to our ability to cope with a constantly changing environment, and understanding their neural bases is thus a key goal of neuroscience. However, most relevant studies have used multidimensional switch tasks, which conflate different switch

Received Nov. 10, 2010; revised Jan. 12, 2011; accepted Feb. 7, 2011.

This study was supported by National Institute on Aging Grant R01 AG033036 and National Science Foundation Grant BCS-0814302. We thank two anonymous reviewers for helpful comments on an earlier version of this manuscript.

Correspondence should be addressed to Dr. Brian T. Gold, Anatomy and Neurobiology, MN 214, Medical Sciences Building, University of Kentucky Medical Center, 800 Rose Street, Lexington, KY 40536-0098. E-mail: brian.gold@uky.edu.

DOI:10.1523/JNEUROSCI.5923-10.2011

Copyright $\odot 2011$ the authors $\quad 0270-6474 / 11 / 314771-09 \$ 15.00 / 0$ types. For example, in the frequently used color-shape task, the two stimulus dimensions are presented simultaneously (e.g., a blue square) and are associated with different S-R mappings. Participants must therefore switch attention between two stimulus dimensions (stimulus switching) while also switching between two S-R mappings that correspond to these different stimulus dimensions (response switching).

Nevertheless, a few neuroimaging studies that have compared different switch types have reported some domain preferentiality in brain activation patterns (Nagahama et al., 2001; Rushworth et al., 2002; Ravizza and Carter, 2008). For example, Ravizza and Carter (2008) found greater dorsolateral PFC (DLPFC) [Brodmann's area (BA) 9/46] activation for response than for perceptual switching and a trend toward greater dorsal premotor cortex (BA 6) activation for perceptual than for response switching. Further, results from a recent meta-analysis found evidence for domain-preferential perceptual-switching activation in the rostral portion of the dorsal premotor cortex (pre-PMd) and for set switching in frontopolar cortex (FPC) (Kim et al., 2011b).

Results from these studies raise the intriguing possibility that multiple brain regions may guide qualitatively different switch processes, with regional specialization depending upon the kind of switch being performed. However, a proper test of this hypothesis requires the use of a single task, in which multiple switch types are performed on a common set of stimuli, and reaction time switch costs are similar across switch types to equate difficulty. These criteria have not been met in previous empirical studies or meta-analyses.

In the present study, we designed a paradigm that selectively induced stimulus, response, and set switches in the context of a single task decision performed on a common set of stimuli to test 
whether multiple brain regions contribute to different types of cognitive flexibility. The use of a rapid event-related design enabled assessment of brain activations associated with correct switch trials, and pilot testing ensured comparable switch costs between conditions.

\section{Materials and Methods}

Subjects. Sixteen healthy subjects between the ages of 20 and 28 years $(10$ females, mean age $=$ $24.1, \mathrm{SD}=2.6$; mean years of education $=$ $15.1, \mathrm{SD}=2.3$ ) participated. All subjects were right-handed, native English speakers who reported no neurological disease and had normal or corrected-to-normal visual acuity. Subjects provided written informed consent in a manner approved by the University of Kentucky Institutional Review Board and were paid for participating.

Task procedure. We designed a task paradigm that allowed us to independently measure three distinct switch types and a non-switch condition, all of which were embedded within a color-digit comparison task. The three switch conditions were stimulus switch (Stim-Sw), response switch (Resp-Sw), and cognitive set switch (Set-Sw) conditions. Subjects received training on the color-digit comparison task before functional magnetic resonance imaging (fMRI) scanning. Prescan training consisted of three different sessions. All stimuli were presented in one of four small boxes, embedded within a larger box.

In the first two sessions, the subjects learned the color-digit pair associations. Importantly, two separate color-digit training sessions were used to induce subjects to form two distinct "cognitive sets." In the first training session (Fig. 1A), subjects learned to associate the color red with the digits 5 or 7 depending on the side of presentation (i.e., left- 5 and right-7). In the second training session (Fig. $1 B$ ), subjects learned to associate the color green with the digits 6 or 4 (i.e., left- 6 and right- 4 ). In these two training sessions, subjects were asked to verbalize the digit associated with the color square presented in a given location. For example, for a red square presented in the left box, subjects were to say "five", and for a green square presented in the right box, subjects were to say "four". In the third session, subjects learned the response rules (Fig. 1C). Here, two digits from 2 to 9 were presented simultaneously within the same box. Subjects were asked to select the larger digit value if the digits were presented in the top row and the smaller digit value if they were presented in the bottom row. For the response rules training session, subjects responded via a left or right button press. Subjects completed a total of 24 trials in each training session.

Stimuli and task conditions used during scanning are illustrated in Figure $1 D$. For all trial types, four spatially contiguous white boxes were presented on a black background with a digit and a color square in one of the boxes. All digits were presented in white, and the square was presented in either red or green. The color-digit comparison task required subjects to: (1) select the appropriate digit set based on the associated color square (i.e., red $=5,7$; green $=6,4$ ) and side of presentation (i.e., a leftward red square $=5$; a rightward red square $=7) ;(2)$ compare it to the simultaneously presented digit; (3) select a correct response rule according to the row (i.e., top = higher digit; bottom = lower digit); and (4) respond via a left or right button press.

Non-Sw used the same color, side, and row as the previous trial, with only the presented digit (and thus correct response) changing from the previous trial. Stim-Sw was cued by a change in the side to which stimuli were presented from the previous trial. The color of the square and the row in which it was located remained the same as in the previous trial. The training session ensured that subjects learned to strongly associate a side of presentation with a specific digit within a cognitive set (a stimulus color). Stim-Sw trials required subjects to switch between two unambiguous perceptual-stimulus associations within a cognitive set (e.g., switch from digit 5 to digit 7 within the red cognitive set because the red square has been moved from the left side to the right side). Thus, Stim-Sw trials were constrained by a perceptual-stimulus association which directly specified the correct comparator digit for the digit-color comparison task. This form of switching can be thought of as switching between "what" levels of internal representation.

Resp-Sw was cued by a change in the row in which stimuli were presented from the previous trial (e.g., from top to bottom). The color of the square and the side in which it was presented remained the same as in the previous trial. Resp-Sw trials required subjects to shift attention between two unambiguous perceptual-cue/response-rule associations within a cognitive set (e.g., switch from the "select the higher digit rule" to "select the lower digit rule" because the red square has moved from the top row to the bottom row). Thus, Resp-Sw trials directly specified the rule to be used for the digit-color comparison task within a cognitive set (the top row indicated the higher digit rule and the bottom row indicated the lower digit rule). However, Resp-Sw was more abstract/conceptual than Stim-Sw because it required the application of a new rule to the digitcolor comparison task as opposed to the use of the same rule with a different digit (as in Stim-Sw trials). This form of switching can be thought of as switching between "how" levels of internal representation (i.e., how to respond).

Set-Sw was cued by a change in the square's color from the previous trial (e.g., from red to green). The stimuli were presented on the same side and row as the previous trial. The training session ensured that subjects learned to strongly associate a color with a set of digits or cognitive set (i.e., red was associated with the 5/7 cognitive set and green was associated with the $6 / 4$ cognitive set). Set-Sw trials required subjects to shift attention from one cognitive set to another (e.g., a change from the red square to a green square indicated that a new digit pair of $6 / 4$ must be considered). Unlike the other switch conditions, a change in the square's color (Set-Sw) did not unambiguously specify the correct comparator digit for the digit-color comparison task because the correct digit had to be retrieved de novo from a new digit set. Set switches thus emphasized endogenous control processes associated with the internal generation and reconfiguration of a new cognitive set. This form of switching can be thought of as switching between "context" levels of internal representation (i.e., the context in which stimulus and response rules are selected).

An event-related design was used, in which different trial types of interest were separated from each other by a variable intertrial interval 
(range $=2.2-8.1 \mathrm{~s}$, mean $=4.15 \mathrm{~s})$ consisting of fixation. To ensure equal numbers of trial types, a pseudorandom presentation order was used such that switch trials were followed by two or more non-switch trials. Across the experiment, there were a total of 52 trials per condition. The experiment was divided into 4 runs, each composed of 13 trials per condition. All stimuli and tasks were generated and programmed via E-Prime 1.2.

Imaging acquisition. Imaging data were collected on a 3 Tesla Siemens TIM scanner at the Magnetic Resonance Imaging and Spectroscopy Center of University of Kentucky. Foam padding was used to limit head motion within the coil. T2*-weighted images were acquired using a gradient-echo echoplanar imaging (EPI) sequence [repetition time $(\mathrm{TR})=2000$, echo time $(\mathrm{TE})=30 \mathrm{~ms}$, flip angle $=77^{\circ}, 33$ axial slices, field of view $(\mathrm{FOV})=224 \times 224$, image matrix $=64 \times 64$, isotropic 3.5 $\mathrm{mm}$ voxels]. A double-echo gradient-echo sequence $(\mathrm{TE} 1=5.19 \mathrm{~ms}$, $\mathrm{TE} 2=7.65 \mathrm{~ms}$ ) with slice position and spatial resolution matching those of the EPI acquisition was used to map the spatial inhomogeneity of the $\mathrm{B}_{0}$ field. T1-weighted structural images were collected using the magnetization-prepared rapid gradient-echo (MPRAGE) sequence $\left(\mathrm{TR}=2100 \mathrm{~ms}, \mathrm{TE}=2.93 \mathrm{~ms}\right.$, inversion time $=1100 \mathrm{~ms}$, flip angle $=12^{\circ}$, FOV $=224 \times 256 \times 192 \mathrm{~mm}, 1 \mathrm{~mm}$ isotropic voxels, sagittal partitions).

fMRI preprocessing and voxelwise analyses. SPM5 (Statistical Parametric Mapping; Wellcome Department of Cognitive Neurology, UCL, London, UK) was used in the preprocessing and statistical analyses of imaging data. Preprocessing of the fMRI data included the following steps. After discarding the first five functional volumes (10 s) of each run, differences in timing between slices were adjusted using the sinc interpolation (Henson et al., 1999). Images were then registered to the first volume of the first session using a six-parameter rigid body transformation. Next, images were unwarped via $B_{0}$ field maps to reduce nonlinear magnetic field distortions. The functional images were then coregistered with the MPRAGE and were normalized into $2 \mathrm{~mm}$ isotropic voxels using the standard MNI T1 brain. Finally, the normalized images were smoothed with an $8 \mathrm{~mm}$ full-width at half-maximum Gaussian kernel and high-pass filtered with a 128 s cutoff period.

For the first-level individual analysis, all experimental trials were used to construct a general linear model using a canonical hemodynamic response function (HRF) with temporal and dispersion derivatives. Head movement parameters in six dimensions, estimated during motion correction, were included in the model as nuisance covariates. Error trials and the first trial of each run were modeled as a separate regressor of non-interest. Consequently, only correct trials were included in the fMRI analyses. Non-Sw was contrasted with Set-Sw, Resp-Sw, and Stim-Sw, resulting in contrast images which were then submitted to the group level analyses.

For second-level group analyses, each of the three switch conditions were compared with Non-Sw. For these analyses, the statistical threshold was corrected at $p<0.05$ level using the false discovery rate (Genovese et al., 2002). A conjunction analysis was then performed using the three contrast images to identify common brain regions involved in all three switch levels. The conjunction analysis tested against the conjunction null (Friston et al., 2005) to identify regions that were commonly activated by each switch condition compared with Non-Sw. Finally, direct comparisons were performed to identify brain regions preferentially associated with each switch type (i.e., Set-Sw vs Resp-Sw and Stim-Sw; Resp-Sw vs Set-Sw and Stim-Sw; and Stim-Sw vs Set-Sw and Resp-Sw). For the conjunction analysis and direct comparisons, uncorrected $p$ values $(p<0.001)$ were used. Only areas of significantly activated clusters with a minimum size of 10 voxels were reported. A conversion tool (Lancaster et al., 2007) was applied to convert MNI coordinates to report peak coordinates in Talairach space (Talairach and Tournoux, 1988).

fMRI region-wise analyses. Time course and magnitude data were extracted from specific PFC regions of interest (ROIs) identified in the voxelwise comparisons described above (the conjunction analysis and the three direct comparisons) for computation of neural switch costs and their correlation with behavioral switch costs. Behavioral switch costs were computed by subtracting the mean reaction times (RTs) of Non-Sw from each of the switch conditions (Set-Sw, RespSw, and Stim-Sw).
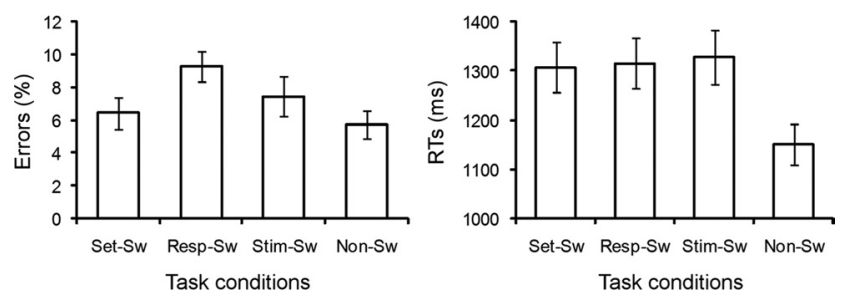

Figure 2. Mean error rates (left) and mean RTs (right) for switch conditions. Error bars represent the SEM.

ROI masks were centered on peak voxelwise activation differences and consisted of a three-dimensional area including all contiguous voxels within $6 \mathrm{~mm}$ of the peak coordinates. A $16 \mathrm{~s}$ blood oxygen leveldependent (BOLD) time course was extracted from each ROI to explore the temporal activation profile associated with each condition. For computation of neural switch costs, the magnitudes of signal changes at the third time point ( $4 \mathrm{~s}$ after stimulus onset) were extracted to capture the peak of the BOLD response. Neural switch costs were then computed for each subject, in each condition, by subtracting subjects' peak BOLD responses in Non-Sw from their peak BOLD responses in each of the switch conditions (Set-Sw, Resp-Sw, and Stim-Sw). Correlations were then run between behavioral switch costs and neural switch costs.

\section{Results}

\section{Behavioral data}

Mean error rates and RTs are presented in Figure 2. Accuracy was uniformly high across conditions (all conditions $\geq 91 \%$ ). Behavioral performance was significantly less accurate for the three switch conditions than Non-Sw $\left(F_{(1,15)}=10.61, p=0.005\right)$. The error rates for Resp-Sw were higher than the two other switch conditions $\left(F_{(1,15)}=12.01, p=0.003\right)$. There was no difference in error rates between Set-Sw and Stim-Sw $\left(F_{(1,15)}=0.86\right.$, $p=0.37)$.

For the analysis of RT data, only correct trials were included in calculating RTs for each experimental condition. RTs were significantly longer for the three switch conditions when compared with Non-Sw $\left(F_{(1,15)}=112.69, p=0.001\right)$. In other words, all three switch conditions showed significant switch costs (set switch cost $=157 \mathrm{~ms}$, response switch cost $=166 \mathrm{~ms}$, and stimulus switch cost $=179 \mathrm{~ms})$. However, there was no significant difference in the RTs among the three switch conditions $\left(F_{(2,30)}=\right.$ $0.40, p=0.67)$. Post hoc analyses confirmed that there were no RT differences in pairwise comparisons between switch costs in the three conditions ( $p$ values $\geq 0.43$ ).

\section{Imaging data}

The results from the individual analyses of each switch type (Stim-Sw, Resp-Sw, and Set-Sw) compared with Non-Sw are shown on a common inflated surface rendering (Fig. 3) and listed in Tables 1, 2-3. Compared with Non-Sw, Stim-Sw (shown in green) resulted in activation in bilateral caudal PFC regions, prominently involving the rostral portion of the pre-PMd (BA 6) and the caudal portion of the dorsal cingulate cortex (cdACC) (BA 24/32). PFC activation for Stim-Sw was also observed at the junction of the precentral sulcus and inferior frontal sulcus [inferior frontal junction (IFJ); BA 6]. Stim-Sw also resulted in activation of posterior regions, including precuneus, cuneus, lingual gyrus, superior and middle occipital gyri (BA 7/18/19), and thalamus.

Compared with Non-Sw, Resp-Sw (shown in blue) resulted in activation in mid-PFC regions, prominently involving bilateral DLPFC (BAs 9 and 46) and the rostral portion of the dorsal cingulate cortex (rdACC) (BA 32). PFC activation for Resp-Sw 
was also observed in IFJ (BA 6). Resp-Sw also resulted in activation of posterior regions, including precuneus (BA 7), middle temporal gyrus (BA 19/39), lingual gyrus (BA 18), and cuneus (BA 17/18).

Compared with Non-Sw, Set-Sw (shown in red) resulted in activation in rostral-PFC regions, prominently involving lateral and medial portions of FPC (BA 10). Other PFC activations for Set-Sw were observed in IFJ (BA 6) and left inferior frontal gyrus (BA 44/45). Set-Sw also resulted in activation of posterior regions, including precuneus (BA 7), superior and inferior parietal lobules (SPL and IPL; BAs 7 and 40), and occipitotemporal areas including the cuneus, lingual gyrus (BA 17/ 18), superior occipital gyrus (BA 19), and middle temporal gyrus (BA 37).

A conjunction analysis was then conducted to identify common areas of activation across Set-Sw, Resp-Sw, and Stim-Sw compared with Non-Sw. The results showed prominent activation of left IFJ (BA 6) and a large cluster within posterior parietal cortex (PPC), extending into the superior occipital gyrus (Fig. 4). The coordinates of these regions, and several other regions showing common activation across switch conditions, are listed in Table 4.

The next analyses focused on direct comparisons between three switch contrasts to identify regions preferentially activated by each switch type (Fig. 5; Table 5). Areas preferentially activated by Stim-Sw (shown in green) compared with Resp-Sw and Set-Sw were the left pre-PMd (BA 6) and cdACC (BA 24). Areas preferentially activated by Resp-Sw (shown in blue) compared with Stim-Sw and Set-Sw were the left DLPFC (BA 9/46) and rdACC (BA 32). Finally, areas preferentially activated by Set-Sw (shown in red) compared with Stim-Sw and Resp-Sw were the left lateral FPC (LFPC) (BA 10) and left medial FPC (MFPC) (BA 10).

In summary, results from the direct comparisons confirmed the pattern of PFC activation observed in the individual analyses of each switch type (described above). Specifically, the direct comparisons demonstrated an anterior-to-posterior gradient of activation across lateral and medial frontal cortex according to switch type. The most anterior activations were observed for Set-Sw (lateral and medial FPC). Resp-Sw resulted in activations situated posterior to Set-Sw activations (DLPFC and rdACC). Finally, Stim-Sw resulted in activations posterior to those of Resp-Sw (pre-PMd and cdACC).

To further explore the strength of switch-type preferentiality of PFC regions identified in the whole-brain analyses, correlations were run between behavioral and neural switch costs. From the conjunction analysis results we calculated neural switch costs in left IFJ $(x, y, z=-50,1,40)$ for each of the three switch conditions. In addition, from the results of the direct comparisons,
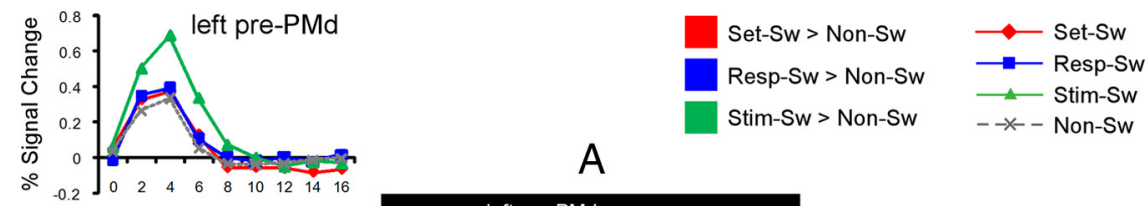

A
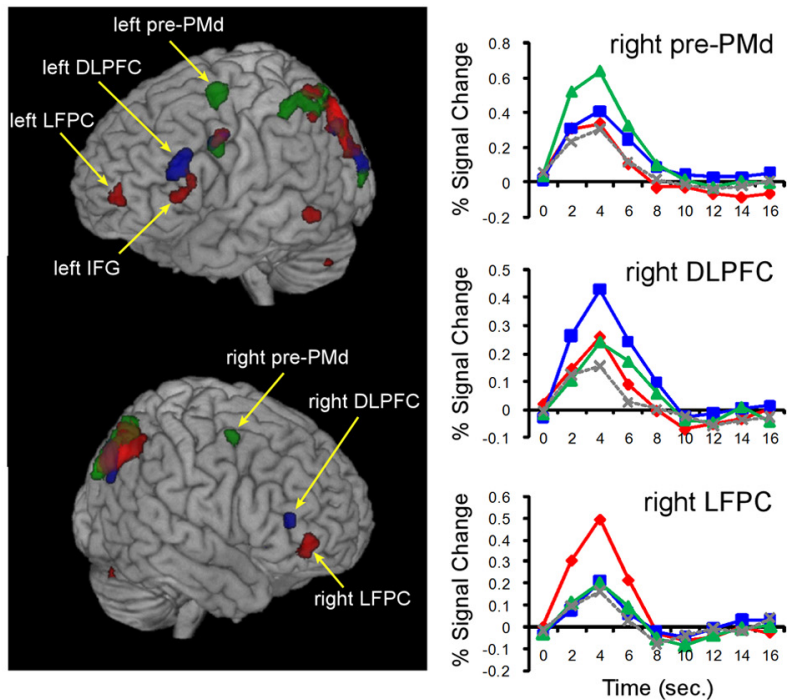

B

Time (sec.)
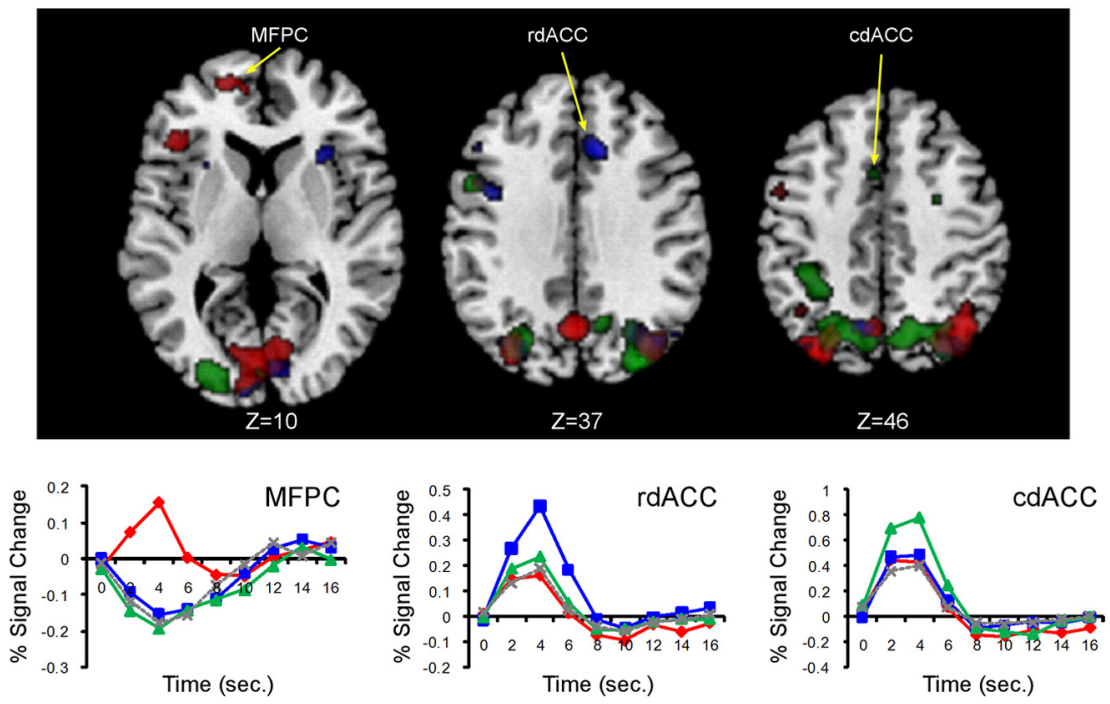

Figure 3. Significant brain activations for each switch type. $\boldsymbol{A}, \boldsymbol{B}$, Activations in the lateral $(\boldsymbol{A})$ and medial $(\boldsymbol{B})$ prefrontal cortex for Stim-Sw (green), Resp-Sw (blue), and Set-Sw (red), compared with Non-Sw. Hemodynamic time courses are presented for prefrontal regions showing preferential activation according to switch type.

neural switch costs were calculated in the following ROIs: left LFPC $(x, y, z=-19,50,16)$, left DLPFC $(x, y, z=-44,26,26)$, left pre-PMd $(x, y, z=-30,-8,60), \operatorname{MFPC}(x, y, z=-10,48,18)$, $\operatorname{rdACC}(x, y, z=6,17,38)$, and $\operatorname{cdACC}(x, y, z=-5,-2,47)$.

Results from the correlation analyses demonstrated a domaingeneral activation pattern of left IFJ, which showed significant positive correlations $(r=0.76-0.79, p<0.001)$ between neural and behavioral switch costs for each switch type (Fig. $4 C$ ). In striking contrast to the domain-general pattern of correlations observed in left IFJ, other PFC regions showed highly preferential correlation patterns between behavioral and neural switch costs (Fig. 5). The neural switch costs in pre-PMd and cdACC tracked selectively with Stim-Sw behavioral costs $(r=0.69$ and $r=0.71$, respectively, $p$ values $<0.01)$. The neural switch costs in DLPFC and rdACC tracked selectively with Resp-Sw behavioral costs 
Table 1. Significant areas of activation for stimulus switching compared with non-switching

\begin{tabular}{llrrrrr}
\hline Region & Hem & $x$ & $y$ & $z$ & BA & z-score \\
\hline cdACC & $\mathrm{L}$ & -5 & 5 & 48 & $24 / 32$ & 3.29 \\
pre-PMd & $\mathrm{L}$ & -26 & -9 & 61 & 6 & 3.70 \\
IFJ & $\mathrm{L}$ & -50 & 3 & 38 & 6 & 3.49 \\
pre-PMd & $\mathrm{R}$ & 28 & -5 & 57 & 6 & 3.47 \\
SOG/MOG/precuneus & $\mathrm{L}$ & -29 & -75 & 18 & 19 & 5.28 \\
Cuneus/precuneus & $\mathrm{R}$ & 26 & -81 & 29 & $7 / 19$ & 4.62 \\
MOG/lingual gyrus & $\mathrm{L}$ & -24 & -84 & 3 & 18 & 3.87 \\
Thalamus & $\mathrm{L}$ & -12 & -18 & 17 & & 3.84 \\
Cerebellum (pyramis) & $\mathrm{R}$ & 21 & -61 & -30 & & 3.41 \\
Cerebellum (uvula/declive) & $\mathrm{L}$ & -8 & -65 & -27 & & 3.83 \\
Cerebellum (declive) & $\mathrm{R}$ & 6 & -67 & -22 & & 3.98 \\
\hline
\end{tabular}

Hem, Hemisphere; L, left; R, right; SOG, superior occipital gyrus; MOG, middle occipital gyrus.

Table 2. Significant areas of activation for response switching compared with non-switching

\begin{tabular}{llrrrrr}
\hline Region & Hem & \multicolumn{1}{c}{$x$} & \multicolumn{1}{c}{$y$} & BA & z-score \\
\hline IFJ & $\mathrm{L}$ & -38 & -2 & 32 & 6 & 3.97 \\
DLPFC & $\mathrm{R}$ & 45 & 38 & 21 & 46 & 3.74 \\
DLPFC & $\mathrm{L}$ & -48 & 24 & 29 & 9 & 4.18 \\
rdACC & $\mathrm{R}$ & 8 & 18 & 37 & 32 & 4.82 \\
Precuneus & $\mathrm{L}$ & -11 & -68 & 46 & 7 & 4.28 \\
Precuneus/MTG & $\mathrm{R}$ & 26 & -72 & 34 & $19 / 39$ & 4.24 \\
Lingual gyrus & $\mathrm{L}$ & -20 & -78 & -9 & 18 & 5.06 \\
Cuneus/MOG & $\mathrm{L}$ & -28 & -74 & 27 & 19 & 4.02 \\
Cuneus & $\mathrm{R}$ & 6 & -83 & 4 & $17 / 18$ & 3.70 \\
Claustrum & $\mathrm{R}$ & 29 & 19 & 7 & & 4.52 \\
Claustrum & $\mathrm{L}$ & -27 & 15 & 7 & & 3.79 \\
Cerebellum (pyramis) & $\mathrm{L}$ & -14 & -64 & -29 & & 3.45 \\
\hline
\end{tabular}

Hem, Hemisphere; L, left; R, right; MTG, middle temporal gyrus; MOG, middle occipital gyrus.

Table 3. Significant areas of activation for cognitive set switching compared with non-switching

\begin{tabular}{llrrrrr}
\hline Region & Hem & \multicolumn{1}{l}{$y$} & & $z$ & BA & z-score \\
\hline IFG & $\mathrm{L}$ & -42 & 16 & 18 & $44 / 45$ & 3.92 \\
LFPC & $\mathrm{L}$ & -21 & 52 & 11 & 10 & 3.67 \\
MFPC & $\mathrm{L}$ & -8 & 50 & 16 & 10 & 3.62 \\
LFPC & $\mathrm{R}$ & 27 & 43 & 9 & 10 & 3.97 \\
IFJ & $\mathrm{L}$ & -50 & -1 & 41 & 6 & 4.36 \\
Precuneus/SOG & $\mathrm{L}$ & -31 & -76 & 33 & 19 & 4.61 \\
Precuneus/SPL & $\mathrm{R}$ & 30 & -74 & 34 & $7 / 19$ & 4.60 \\
Precuneus & $\mathrm{R}$ & 7 & -69 & 52 & 7 & 4.08 \\
SPL & $\mathrm{L}$ & -32 & -61 & 54 & 7 & 3.46 \\
IPL & $\mathrm{L}$ & -41 & -60 & 41 & 40 & 3.29 \\
MTG & $\mathrm{L}$ & -57 & -49 & -9 & 37 & 3.51 \\
Precuneus/PCC & $\mathrm{L}$ & 0 & -64 & 27 & $7 / 30 / 31$ & 4.69 \\
Cuneus/lingual gyrus & $\mathrm{R}$ & 1 & -77 & 8 & $17 / 18$ & 4.56 \\
Cerebellum (tuber) & $\mathrm{R}$ & 31 & -66 & -30 & & 3.93 \\
Cerebellum (cerebellar tonsil) & $\mathrm{L}$ & -36 & -60 & -38 & & 3.64 \\
\hline
\end{tabular}

Hem, Hemisphere; L, left; R, right; IFG, inferior frontal gyrus; SOG, superior occipital gyrus; MTG, middle temporal gyrus; PCC, posterior cingulate cortex.

( $r=0.66$ and $r=0.65$, respectively, $p$ values $<0.01$ ). Finally, the neural switch costs in MFPC and LFPC tracked selectively with Set-Sw behavioral costs $(r=0.64$ and $r=0.65$, respectively, $p$ values $<0.01$ ).

\section{Discussion}

The present study dissociated spatially discrete regions of PFC that contribute to domain-general and domain-specific forms of switching using a common task and set of stimuli. Importantly, RT switch costs were not different between conditions and each of the regions characterized as domain preferential displayed activation patterns which were selectively correlated with the behavioral costs of only one switch type. These findings suggest that preferential activation for specific switch types were unlikely to result from differences in generic cognitive effort. Results demonstrate that multiple PFC regions show cognitive flexibility, with regional specialization depending upon the kind of flexibility required. At the broadest level, results suggest a rostrocaudal gradient across the lateral and medial PFC according to the degree of representational abstraction engendered by an act of cognitive flexibility.

\section{Domain-general switching mechanisms within the frontoparietal network}

Prominent activation common to each switch type was observed in IFJ and PPC. The IFJ is a posterior lateral region of frontal cortex near the junction of the inferior frontal sulcus and the inferior precentral sulcus ( $\sim \mathrm{BA} 44 / 6 / 9)$. The PPC comprises a wide expanse of parietal cortex $(\sim \mathrm{BA} 7 / 40)$, including much of the inferior and superior parietal lobules. This finding suggests that IFJ and PPC contribute core cognitive processes generic to task switching. Two cognitive processes which are thought to contribute to all forms of switching are representing and updating task sets (Miyake et al., 2000), making IFJ and PPC potential contributors to these domain-general switch processes.

If this were the case, then two expectations should follow. First, there should be existing evidence supporting a role for IFJ and PPC in representing and updating task sets from previous studies. There is support for this expectation. Specifically, previous studies have implicated IFJ in updating task rules or sets across a range of cognitive control tasks (Brass and von Cramon, 2004; Derrfuss et al., 2004, 2005; Roth et al., 2006, 2009; Roth and Courtney, 2007). Analogously, there is evidence of a role for PPC in representing task sets (Bunge et al., 2002, 2003; Cavina-Pratesi et al., 2006).

The second expectation concerns the intimate relationship between the cognitive processes of representing and updating task sets, which suggests that there should be a coordinated role of regions which putatively support these processes (i.e., IFJ and PPC). Support for this expectation comes from diffusion tensor imaging (DTI) tractography studies, which have demonstrated that portions of the IFJ ( $\sim$ BA 44/6) and PPC ( $\sim$ BA 40/7) are anatomically connected via the superior longitudinal fasciculus (SLF) (Catani et al., 2005). Further, we recently found that the strength of anatomical connectivity (assessed via DTI metric of fractional anisotropy) along the SLF tract is negatively correlated with switch cost RT in young and older adults (Gold et al., 2010). This suggests that faster task switching is associated with "more direct" information flow between IFJ and PPC, consistent with a view that these regions play a coordinated role during switching.

\section{Anterior prefrontal cortex supports cognitive set switching}

Cognitive Set-Sw preferentially recruited portions of lateral and medial FPC, a finding which is consistent with a previously known role for FPC in the internal generation of cognitive representations. For example, FPC has been associated with planning (Koechlin et al., 1999, 2000; van den Heuvel et al., 2003), envisioning/predicting future events (Partiot et al., 1995; Okuda et al., 2003), reasoning (Christoff et al., 2001; Kroger et al., 2002), maintaining rules guiding subsequent cognitive activity (Sakai and Passingham, 2006), and endogenous set switching (Rogers et al., 2000; Weidner et al., 2002). 
At first pass, it may appear that preferential FPC activation for Set-Sw could relate to episodic memory retrieval demands since Set-Sw induced subjects to retrieve a previously learned digit set. However, Stim-Sw also required episodic retrieval. Specifically, Stim-Sw required retrieval of a specific digit within a set. Results from episodic retrieval studies suggest that FPC activity tends to increase when a specific aspect of an item must be retrieved (Ranganath et al., 2000). Thus, increased episodic retrieval demands do not appear to account for the preferential FPC activation we observed during Set-Sw.

\section{Mid-prefrontal regions support response switching}

Resp-Sw preferentially recruited left DLPFC (BAs 9 and 46) and the rostral portion of the dorsal cingulate cortex (rdACC; BA 32). This finding is consistent with several recent studies which have directly contrasted perceptual-based and responsebased cognitive control processes. For example, Ravizza and Carter (2008) found greater DLPFC activity for response switching than for perceptual switching. Similarly, in our recent work using a modified version of the Stroop task, DLPFC was activated during response conflict but not perceptual conflict (Kim et al., 2010, 2011a).

The present results demonstrate a preferential role for medial PFC (rdACC), in addition to lateral PFC (DLPFC), in Resp-Sw. One difference between the present Resp-Sw and those in some previous studies (Ravizza and Carter, 2008) is that the present condition involved switching between response-rules (a conceptual-level representational shift) rather than simple S-R mappings. Conflict monitoring theory suggests that rdACC contributes to conflict detection whereas DLPFC contributes to conflict resolution at the response level (Botvinick et al., 1999, 2001; MacDonald et al., 2000). It is thus possible that response rule switches enhance conflict detection mechanisms compared with $\mathrm{S}-\mathrm{R}$ switches, which could drive the activation of rdACC during response rule switching.

\section{Posterior prefrontal regions support stimulus switching}

Stim-Sw preferentially recruited pre-PMd (BA 6) and cdACC (BA 24). The pre-PMd region comprises a rostral portion of premotor cortex and is more heavily interconnected with PFC than with motor cortex (Barbas and Pandya, 1987). Data from a body of lesion studies in monkeys and humans suggest that pre-PMd's role in stimulus-motor relationships relates to learning arbitrary perceptual-stimulus associations (Petrides, 2005; Amiez et al., 2006). Based on such evidence, an emerging theory is that prePMd is involved in learning and applying rule-based associations between perceptual features of stimuli and responses (Badre and D'Esposito, 2009). The present results demonstrate that prePMd is actively involved in switching between internal representations per se and does so in a domain-general manner. In other words, the same region that contributes the basic representational building blocks to learning arbitrary perceptual-stimulus
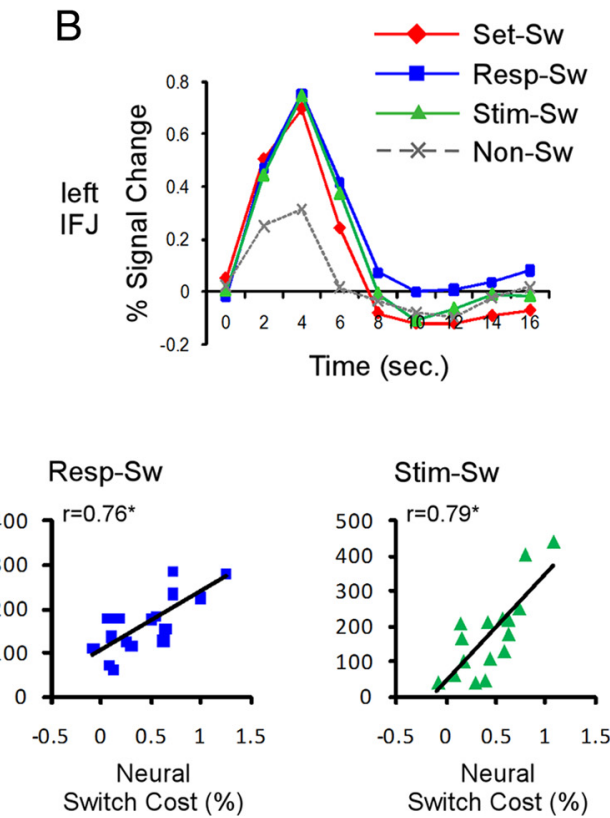

Table 4. Common areas of activation across switch types compared with non-switching

\begin{tabular}{llrrrll}
\hline Region & Hem & \multicolumn{1}{l}{$x$} & $y$ & $z$ & BA & z-score \\
\hline IFJ & $\mathrm{L}$ & -50 & 1 & 40 & 6 & 3.74 \\
PPC/SOG & $\mathrm{L}$ & -31 & -74 & 29 & $7 / 19$ & 4.84 \\
Precuneus & $\mathrm{L}$ & -11 & -74 & 49 & 7 & 3.76 \\
Precuneus/SPL & $\mathrm{R}$ & 28 & -74 & 34 & $7 / 19$ & 4.82 \\
Cuneus & $\mathrm{L}$ & -9 & -81 & 9 & $17 / 18$ & 3.77 \\
\hline
\end{tabular}

Hem, Hemisphere; L, left; R, right; SOG, superior occipital gyrus.

associations also supports switching between representations of these associations.

\section{Anterior-posterior PFC switching gradient associated with endogenous control}

Results suggest an anterior-posterior gradient across PFC, according to the degree of endogenous control required by a task switch. Progressively more rostral regions were recruited as switches became increasingly abstract and required more endogenous control. The most anterior activations were observed for Set-Sw, for which perceptual cues did not unambiguously specify the correct comparator digit for the task because the correct digit had to be retrieved de novo from a new digit set. Set-Sw is thus thought to emphasize endogenous control processes associated with the internal generation and maintenance of task sets within working memory (Dreher et al., 2002).

In contrast, the most posterior activations (pre-PMd and cdACC) were observed for Stim-Sw, which can be viewed as relatively low in the need for endogenous control because external switch cues directly specify the dimension of focus (Koch, 2003). Located spatially in between activations for Set-Sw and Stim-Sw were Resp-Sw activations in mid-PFC regions (DLPFC and rdACC). Like Stim-Sw trials, Resp-Sw trials directly specified the rule to be used for the digit-color comparison task within a cognitive set. However, Resp-Sw in the present experiment was more abstract than Stim-Sw because it required a change in the rule for 

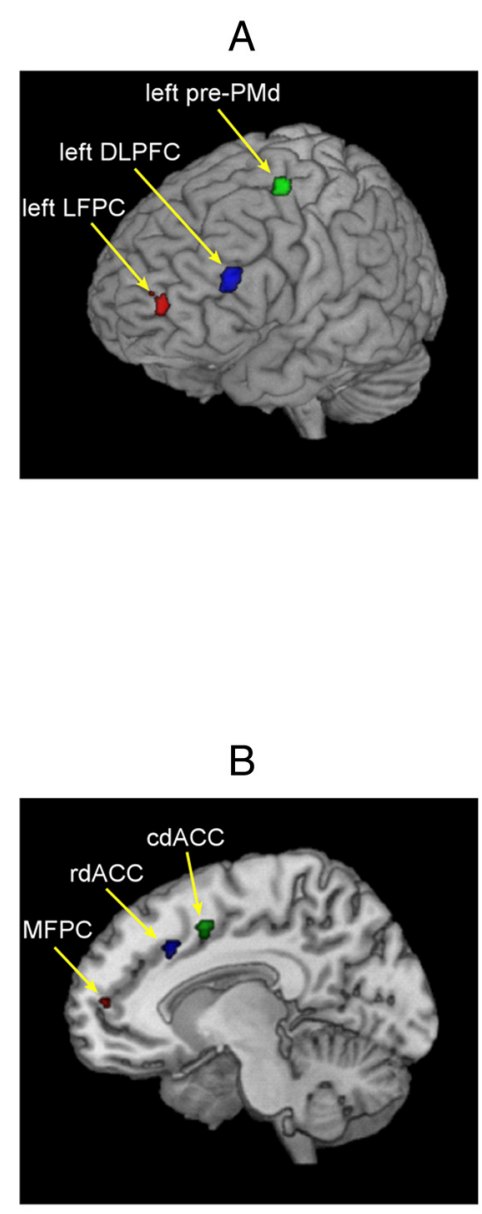

Set-Sw
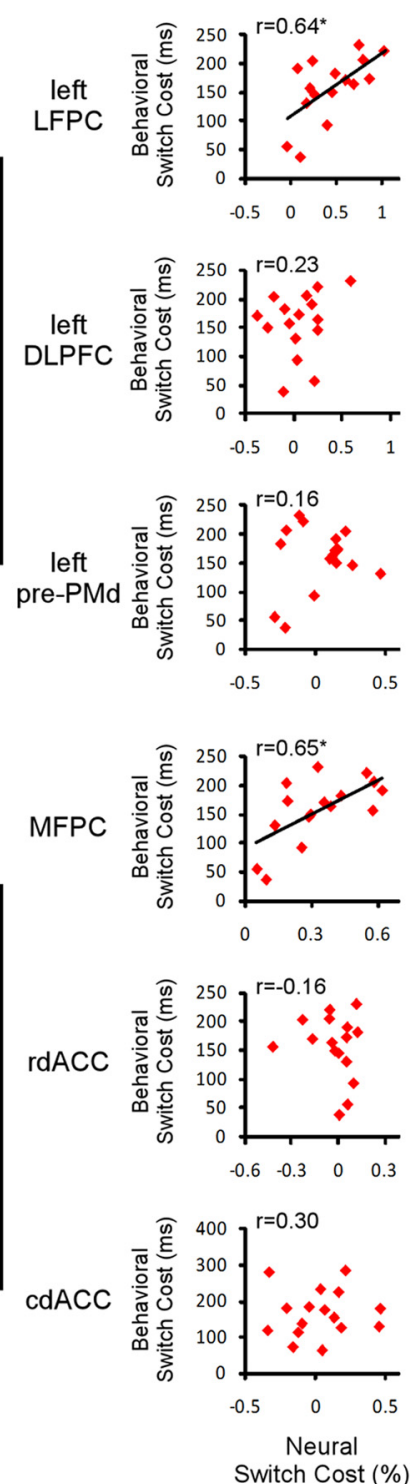

Resp-Sw
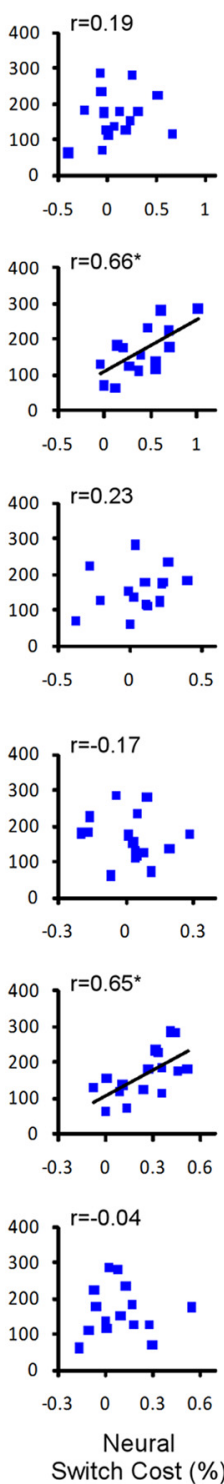

Stim-Sw
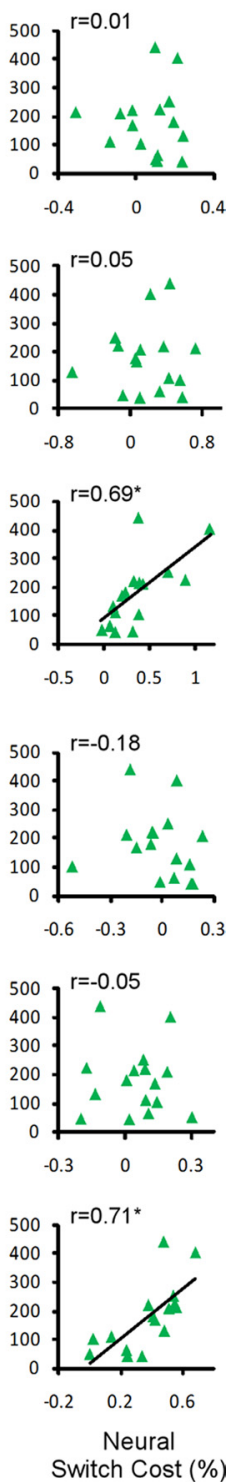

Figure 5. $\quad \boldsymbol{A}, \boldsymbol{B}$, Preferential areas of activation in the left lateral $(\boldsymbol{A})$ and medial ( $\boldsymbol{B})$ PFC for Set-Sw, Resp-Sw, and Stim-Sw. Correlations between behavioral and neural switch costs for three switch types within selected ROls are presented. The asterisks denote statistical significance $(p<0.01)$.

Table 5. Preferential areas of activation for different switch types

\begin{tabular}{lllllll}
\hline Region & Hem. & $x$ & $y$ & $z$ & BA & $z$-score \\
\hline Cognitive set switching & & & & & & \\
$\quad$ LFPC & $\mathrm{L}$ & -19 & 50 & 16 & 10 & 3.53 \\
$\quad$ MFPC & $\mathrm{L}$ & -10 & 48 & 18 & 10 & 3.92 \\
$\begin{array}{l}\text { Response switching } \\
\quad \text { DLPFC }\end{array}$ & $\mathrm{L}$ & -44 & 26 & 26 & $9 / 46$ & 4.17 \\
$\quad$ rdACC & $\mathrm{R}$ & 6 & 17 & 38 & 32 & 4.38 \\
$\begin{array}{l}\text { Stimulus switching } \\
\quad \text { pre-PMd }\end{array}$ & $\mathrm{L}$ & -30 & -8 & 60 & 6 & 3.91 \\
$\quad$ cdACC & $\mathrm{L}$ & -5 & -2 & 47 & 24 & 3.60 \\
\hline
\end{tabular}

Hem, Hemisphere; L, left; R, right.

responding to the task (a conceptual-level representational shift) as opposed to the application of the same rule to a different stimulus (i.e., a change in "how" rather than "what" levels of internal representation).

Our findings are partly consistent with the hierarchical organization model of PFC function (Koechlin et al., 2003). In particular, the present results concur with the hierarchical model that rostral PFC regions are involved in control operations related to the selection of task sets of $\mathrm{S}-\mathrm{R}$ associations according to ongoing internal goals, whereas more posterior $\mathrm{PFC}$ regions are involved in sensory control processes in response to external cues. However, the hierarchical organization model holds that "lower-level" regions (e.g., premotor cortex) are dependent on the operation of "higher-level" regions (e.g., DLPFC). In contrast, the present results suggest that, within the domain of task switching, some posterior PFC regions (e.g., pre-PMd) appear to be capable of guiding task switches in the absence of prominent involvement of more anterior PFC regions.

The present anterior-to-posterior PFC task-switching gradient is similar to a previously reported gradient in "representational abstraction" that has been observed in language and verbal memory domains across ventrolateral PFC (Gold and Buckner, 2002; Badre et al., 2005; Gold et al., 2006). Our results support a view that there exists a parallel functional organization across 
lateral and medial PFC according to the level of abstraction involved in cognitive control (Banich, 2009; Egner, 2009; Kouneiher et al., 2009). The existence of multiple qualitatively distinct switch mechanisms within PFC likely contributes to our ability to flexibly adjust to our environment on a moment-to-moment basis.

\section{References}

Amiez C, Kostopoulos P, Champod AS, Petrides M (2006) Local morphology predicts functional organization of the dorsal premotor region in the human brain. J Neurosci 26:2724-2731.

Badre D, D'Esposito M (2009) Is the rostro-caudal axis of the frontal lobe hierarchical? Nat Rev Neurosci 10:659-669.

Badre D, Poldrack RA, Paré-Blagoev EJ, Insler RZ, Wagner AD (2005) Dissociable controlled retrieval and generalized selection mechanisms in ventrolateral prefrontal cortex. Neuron 47:907-918.

Banich MT (2009) Executive function: the search for an integrated account. Curr Dir Psychol Sci 18:89-94.

Barbas H, Pandya DN (1987) Architecture and frontal cortical connections of the premotor cortex (area 6) in the rhesus monkey. J Comp Neurol 256:211-228.

Botvinick M, Nystrom LE, Fissell K, Carter CS, Cohen JD (1999) Conflict monitoring versus selection-for-action in anterior cingulate cortex. Nature 402:179-181.

Botvinick MM, Braver TS, Barch DM, Carter CS, Cohen JD (2001) Conflict monitoring and cognitive control. Psychol Rev 108:624-652.

Brass M, von Cramon DY (2004) Decomposing components of task preparation with functional magnetic resonance imaging. J Cogn Neurosci 16:609-620.

Bunge SA, Hazeltine E, Scanlon MD, Rosen AC, Gabrieli JD (2002) Dissociable contributions of prefrontal and parietal cortices to response selection. Neuroimage 17:1562-1571.

Bunge SA, Kahn I, Wallis JD, Miller EK, Wagner AD (2003) Neural circuits subserving the retrieval and maintenance of abstract rules. J Neurophysiol 90:3419-3428.

Catani M, Jones DK, ffytche DH (2005) Perisylvian language networks of the human brain. Ann Neurol 57:8-16.

Cavina-Pratesi C, Valyear KF, Culham JC, Köhler S, Obhi SS, Marzi CA, Goodale MA (2006) Dissociating arbitrary stimulus-response mapping from movement planning during preparatory period: evidence from event-related functional magnetic resonance imaging. J Neurosci 26:2704-2713.

Christoff K, Prabhakaran V, Dorfman J, Zhao Z, Kroger JK, Holyoak KJ, Gabrieli JD (2001) Rostrolateral prefrontal cortex involvement in relational integration during reasoning. Neuroimage 14:11361149.

Derrfuss J, Brass M, von Cramon DY (2004) Cognitive control in the posterior frontolateral cortex: evidence from common activations in task coordination, interference control, and working memory. Neuroimage 23:604-612.

Derrfuss J, Brass M, Neumann J, von Cramon DY (2005) Involvement of the inferior frontal junction in cognitive control: meta-analyses of switching and Stroop studies. Hum Brain Mapp 25:22-34.

Dreher JC, Koechlin E, Ali SO, Grafman J (2002) The roles of timing and task order during task switching. Neuroimage 17:95-109.

Egner T (2009) Prefrontal cortex and cognitive control: motivating functional hierarchies. Nat Neurosci 12:821-822.

Friston KJ, Penny WD, Glaser DE (2005) Conjunction revisited. Neuroimage 25:661-667.

Genovese CR, Lazar NA, Nichols T (2002) Thresholding of statistical maps in functional neuroimaging using the false discovery rate. Neuroimage 15:870-878.

Gold BT, Buckner RL (2002) Common prefrontal regions coactivate with dissociable posterior regions during controlled semantic and phonological tasks. Neuron 35:803-812.

Gold BT, Balota DA, Jones SJ, Powell DK, Smith CD, Andersen AH (2006) Dissociation of automatic and strategic lexical-semantics: functional magnetic resonance imaging evidence for differing roles of multiple frontotemporal regions. J Neurosci 26:6523-6532.
Gold BT, Powell DK, Xuan L, Jicha GA, Smith CD (2010) Age-related slowing of task switching is associated with decreased integrity of frontoparietal white matter. Neurobiol Aging 31:512-522.

Henson RN, Buchel C, Josephs O, Friston KJ (1999) The slice-timing problem in event-related fMRI. Neuroimage 9:125.

Kim C, Chung C, Kim J (2010) Multiple cognitive control mechanisms associated with the nature of conflict. Neurosci Lett 476:156-160.

Kim C, Kroger JK, Kim J (2011a) A functional dissociation of conflict processing within anterior cingulate cortex. Hum Brain Mapp 32: 304-312.

Kim C, Cilles SE, Johnson NF, Gold BT (2011b) Domain general and domain preferential brain regions associated with different types of task switching: a meta-analysis. Hum Brain Mapp. Advance online publication. doi:10.1002/hbm.21199.

Koch I (2003) The role of external cues for endogenous advance reconfiguration in task switching. Psychon Bull Rev 10:488-492.

Koechlin E, Basso G, Pietrini P, Panzer S, Grafman J (1999) The role of the anterior prefrontal cortex in human cognition. Nature 399: $148-151$.

Koechlin E, Corrado G, Pietrini P, Grafman J (2000) Dissociating the role of the medial and lateral anterior prefrontal cortex in human planning. Proc Natl Acad Sci U S A 97:7651-7656.

Koechlin E, Ody C, Kouneiher F (2003) The architecture of cognitive control in the human prefrontal cortex. Science 302:1181-1185.

Kouneiher F, Charron S, Koechlin E (2009) Motivation and cognitive control in the human prefrontal cortex. Nat Neurosci 12:939-945.

Kroger JK, Sabb FW, Fales CL, Bookheimer SY, Cohen MS, Holyoak KJ (2002) Recruitment of anterior dorsolateral prefrontal cortex in human reasoning: a parametric study of relational complexity. Cereb Cortex 12:477-485.

Lancaster JL, Tordesillas-Gutiérrez D, Martinez M, Salinas F, Evans A, Zilles K, Mazziotta JC, Fox PT (2007) Bias between MNI and Talairach coordinates analyzed using the ICBM-152 brain template. Hum Brain Mapp 28:1194-1205.

MacDonald AW 3rd, Cohen JD, Stenger VA, Carter CS (2000) Dissociating the role of the dorsolateral prefrontal and anterior cingulate cortex in cognitive control. Science 288:1835-1838.

Meiran N, Marciano H (2002) Limitations in advance task preparation: switching the relevant stimulus dimension in speeded same-different comparisons. Mem Cognit 30:540-550.

Miller EK, Cohen JD (2001) An integrative theory of prefrontal cortex function. Annu Rev Neurosci 24:167-202.

Miyake A, Friedman NP, Emerson MJ, Witzki AH, Howerter A, Wager TD (2000) The unity and diversity of executive functions and their contributions to complex "frontal lobe" tasks: a latent variable analysis. Cogn Psychol 41:49-100.

Nagahama Y, Okada T, Katsumi Y, Hayashi T, Yamauchi H, Oyanagi C, Konishi J, Fukuyama H, Shibasaki H (2001) Dissociable mechanisms of attentional control within the human prefrontal cortex. Cereb Cortex 11:85-92.

Okuda J, Fujii T, Ohtake H, Tsukiura T, Tanji K, Suzuki K, Kawashima R, Fukuda H, Itoh M, Yamadori A (2003) Thinking of the future and past: the roles of the frontal pole and the medial temporal lobes. Neuroimage 19:1369-1380.

Partiot A, Grafman J, Sadato N, Wachs J, Hallett M (1995) Brain activation during the generation of non-emotional and emotional plans. Neuroreport 6:1397-1400.

Petrides M (2005) Lateral prefrontal cortex: architectonic and functional organization. Philos Trans R Soc B Biol Sci 360:781-795.

Ranganath C, Johnson MK, D’Esposito M (2000) Left anterior prefrontal activation increases with demands to recall specific perceptual information. J Neurosci 20:RC108(1-5).

Ravizza SM, Carter CS (2008) Shifting set about task switching: behavioral and neural evidence for distinct forms of cognitive flexibility. Neuropsychologia 46:2924-2935.

Rogers RD, Andrews TC, Grasby PM, Brooks DJ, Robbins TW (2000) Contrasting cortical and subcortical activations produced by attentional-set shifting and reversal learning in humans. J Cogn Neurosci 12:142-162.

Roth JK, Courtney SM (2007) Neural system for updating object working 
memory from different sources: sensory stimuli or long-term memory. Neuroimage 38:617-630.

Roth JK, Serences JT, Courtney SM (2006) Neural system for controlling the contents of object working memory in humans. Cereb Cortex 16:1595-1603.

Roth JK, Johnson MK, Raye CL, Constable RT (2009) Similar and dissociable mechanisms for attention to internal versus external information. Neuroimage 48:601-608.

Rushworth MF, Hadland KA, Paus T, Sipila PK (2002) Role of the human medial frontal cortex in task switching: a combined fMRI and TMS study. J Neurophysiol 87:2577-2592.

Sakai K (2008) Task set and prefrontal cortex. Annu Rev Neurosci 31:219-245.
Sakai K, Passingham RE (2006) Prefrontal set activity predicts rule-specific neural processing during subsequent cognitive performance. J Neurosci 26:1211-1218.

Talairach J, Tournoux P (1988) Co-planar stereotaxic atlas of the human brain. New York: Thieme.

van den Heuvel OA, Groenewegen HJ, Barkhof F, Lazeron RH, van Dyck R, Veltman DJ (2003) Frontostriatal system in planning complexity: a parametric functional magnetic resonance version of Tower of London task. Neuroimage 18:367-374.

Weidner R, Pollmann S, Müller HJ, von Cramon DY (2002) Top-down controlled visual dimension weighting: an event-related fMRI study. Cereb Cortex 12:318-328. 